\title{
Thermo-oxidative degradation of graphite/epoxy composite laminates: Modeling and long-term predictions
}

\author{
O. Ozcelik, L. Aktas, M. C. Altan* \\ School of Aerospace and Mechanical Engineering, The University of Oklahoma, Norman, OK 73019 USA
}

Received 19 July 2009; accepted in revised form 23 September 2009

\begin{abstract}
Thermo-oxidative degradation of graphite/epoxy composite laminates due to exposure to elevated temperatures was characterized using weight loss and short beam strength (SBS) reduction data. Test specimens obtained from 24-ply, unidirectional AS4/3501-6 graphite/epoxy laminates were subjected to $100,150,175$, and $200^{\circ} \mathrm{C}$ for 5000 hours (208 days) in air. Predictive differential models for the weight loss and short beam strength reduction were developed using the isothermal degradation data only up to 2000 hours. Then, the predictive capabilities of both models were demonstrated using the longer term, 5000 hours degradation data. The proposed models were first order differential expressions that can be used to predict degradation in an arbitrary, time-dependent temperature environment. Both models were able to estimate the actual degradation levels accurately. In particular, excellent agreement was obtained when the degradation temperature was lower than $200^{\circ} \mathrm{C}$.
\end{abstract}

Keywords: polymer composites, graphitelepoxy laminates, thermo-oxidative degradation, short beam strength

\section{Introduction}

Despite their low density and excellent mechanical properties, fiber reinforced polymeric composites (FRPCs) are known to be sensitive to environmental factors. In particular, elevated temperatures coupled with oxygen-induced aging can lead to significant reduction in properties as well as a considerable mass loss. These nonreversible changes are frequently referred to as "thermo-oxidative aging' and are initiated by the diffusion of oxygen into the composite. Elevated temperatures accelerate the oxygen diffusion rate and often lead to oxidation reactions that result in weight loss, microcracks in the resin and resin-fiber debonding [1-3].

Starting with the studies of Greer [4], Nelson [5], and Bowles and Meyers [6], various effects of thermo-oxidative aging on FRPCs have been investigated during the last two to three decades. Obvi- ously, a major goal of such studies is the prediction of long-term degradation behavior and service life of the FRPCs when they are exposed to an arbitrary, time dependent temperature cycle. It is highly impractical to expect full characterization of longterm aging solely by experiments as the time required to carry out such studies is often prohibitive [7]. The modeling efforts have been mostly accomplished by following the changes in one or more degradation parameters such as weight loss, microcrack density or a relevant mechanical property under isothermal conditions. It was also proposed that using the so-called equivalent property time and equivalent cycle time concepts [8-10], time required to achieve a certain degradation state at one temperature can be used to estimate the time required to induce the same degradation at another temperature. Another method to extend the utility of shorter term experiments is to perform these 
experiments at high pressures, thus accelerating the rate of oxygen diffusion without altering the temperature sensitive oxidation reaction kinetics $[2$, 11]. More recently, researchers have tried to establish the link between the degradation kinetics and reduction in mechanical properties in an effort to improve the applicability and predictive capability of aging models $[12,13]$. However, to the best of authors' knowledge, the elusive goal of linking diffusive-oxidative phenomenon to changes in mechanical properties of polymeric composites has not been achieved yet.

In this study, the applicability of first order, differential weight loss and strength reduction models beyond the time frame of the data used to develop these models was investigated. This approach would save significant time provided that an acceptable accuracy can be achieved by only using the shorter term data.

\section{Material and experimental procedure}

Twenty-four-ply, unidirectional, graphite/epoxy (i.e., Hercules AS4/3501-6, Hexcel, Connecticut, USA) laminates were used in this study. The Polyacrylonitrile-based (PAN-based) continuous AS4 fibers have a carbon content of $94 \%$ [14]. The 3501-6 is a Bisphenol A Diglycidyl Ether (DGEBA) type epoxy resin that has been frequently used in aerospace industry [15]. The detailed composition of 3501-6 has been analyzed by Hou and Bai [16]. The composite material system is expected to be used at temperatures up to the recommended cure temperature of $177^{\circ} \mathrm{C}\left(350^{\circ} \mathrm{F}\right)$ [14]. The laminates in this study were cured in an autoclave using a heat rate of $5^{\circ} \mathrm{C} / \mathrm{min}$ followed by a 3 hours hold at $177^{\circ} \mathrm{C}\left(350^{\circ} \mathrm{F}\right)$ at $206.8 \mathrm{kPa}$ (30 psi) pressure. After the fabrication, short beam strength (SBS) test specimens were prepared as suggested by ASTM D 2344-00. All specimens were subsequently dried in a vacuum oven at $60^{\circ} \mathrm{C}$ for $8 \sim 10$ days and kept in a vacuum desiccator until the aging experiments. During aging experiments, the specimens were exposed to four different temperatures: $100,150,175$, and $200^{\circ} \mathrm{C}$ for up to 5000 hours (208 days) in air. Effect of thermooxidative aging was tracked by monitoring the changes in short beam strength and weight of the specimens. Short beam strength values were calculated in three-point bending test mode. As recom- mended by ASTM, test span to sample thickness ratio has to be preferably around 4 to obtain accurate short beam strength values. Since the average thickness of the 24-ply samples was around $3.5 \mathrm{~mm}$, a span of $14 \mathrm{~mm}$ is used to ensure failure due to interlaminar shear stresses. During the course of aging, groups of specimens were removed from the heating chambers at pre-determined times for strength and weight measurements. The threepoint bending tests were carried out at ambient temperature between 20 to $22^{\circ} \mathrm{C}$. The sample deflection during testing was recorded by a linear variable differential transformer (LVDT) system, yielding repeatable results within $0.1 \%$ of the full deflection scale. In each bending tests, 8 samples were used to minimize the experimental uncertainty.

More than 400 composite samples were tested to develop the models. It should be noted that strength reduction models need to accurately capture the initial rate of degradation where the composites experience the highest rate of strength loss. To achieve this, samples need to be tested more frequently in the early stages of the experiments. In fact, modeling short beam strength degradation is critical for estimating service life of composites as it is more sensitive to thermo-oxidative aging due to its strong dependence on matrix properties. Other mechanical properties such as modulus, tensile or flexural strength are influenced more by the fiber properties, and thus experience moderate degradation compared to short beam strength.

\section{Thermo-oxidative degradation results}

\subsection{Short term degradation}

Percentage weight loss experienced by the composite samples during the first 2000 hours of aging at $100,150,175$, and $200^{\circ} \mathrm{C}$ is shown in Figure 1. The temporal evolution of weight loss for each temperature is obtained by weighing the same group of eight samples. Error bars shown in Figure 1 are determined by using $95 \%$ confidence interval. The samples are weighed as frequent as necessary to identify a well established trend such that a differential model, which depends on the time rate of change of weight loss, can be developed. Thus, the weight loss of samples subjected to $200^{\circ} \mathrm{C}$ are measured seven times within the first 250 hours, whereas those exposed to $100^{\circ} \mathrm{C}$ are only measured three times. Figure 1 indicates that at $100^{\circ} \mathrm{C}$, the 


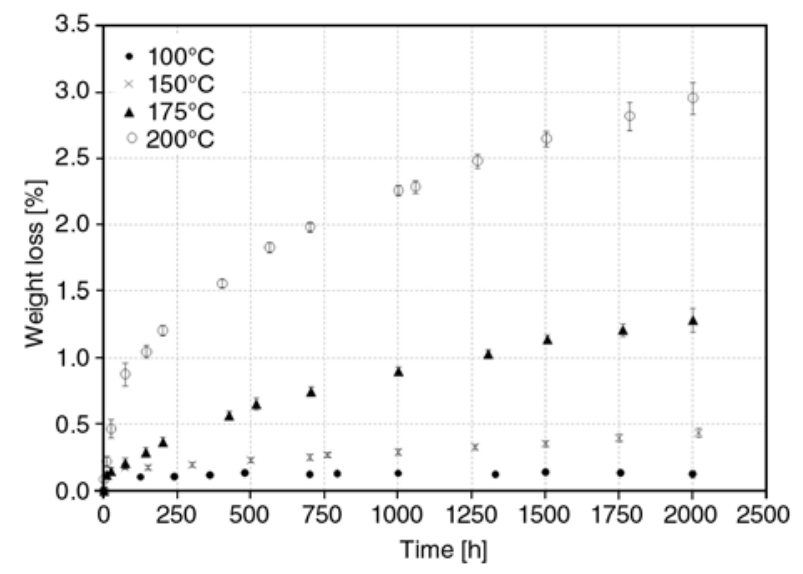

Figure 1. Percentage weight loss of the composite specimens subjected to isothermal thermo-oxidative aging at four different temperatures

samples experienced less than $0.2 \%$ weight loss which remained constant after the first 100 hours of aging. This finite and very small weight loss is possibly due to desorption of moisture that was already in the samples. The fact that the weight loss have not increased with time corroborates this assessment. Samples exposed to 175 and $200^{\circ} \mathrm{C}$ show higher rates of weight loss within the first 500 hours. Rates of weight loss reduced considerably after 1000 hours of aging. Since the same group of eight composite samples was used for each temperature, the variation of percent weight loss at a particular time is very small within a sample group. Hence, the error bars shown in Figure 1 are very small, indicating minimal experimental uncertainty. It is important to point out that the glass transition temperature, $T_{g}$, of this composite laminate is less than $200^{\circ} \mathrm{C}$, most likely between 190 to $195^{\circ} \mathrm{C}$ when it is fully cured $[14,17]$. Thus thermo-oxidative aging at $200^{\circ} \mathrm{C}$ is slightly above glass transition such that the degradation is taking place in the rubbery phase. Clearly, these composite laminates will not be designed to function above glass transition; however, they might experience unexpected overheating or other thermal events that may raise the temperature temporarily above their glass transition. It is very desirable to assess the structural health and the damage sustained by the composite part after such an event. Investigating the effect of an aging temperature above the glass transition is especially meaningful as the possibility of having a single predictive model - as opposed to having separate models for below and above glass transition for a wider temperature range is very appealing.

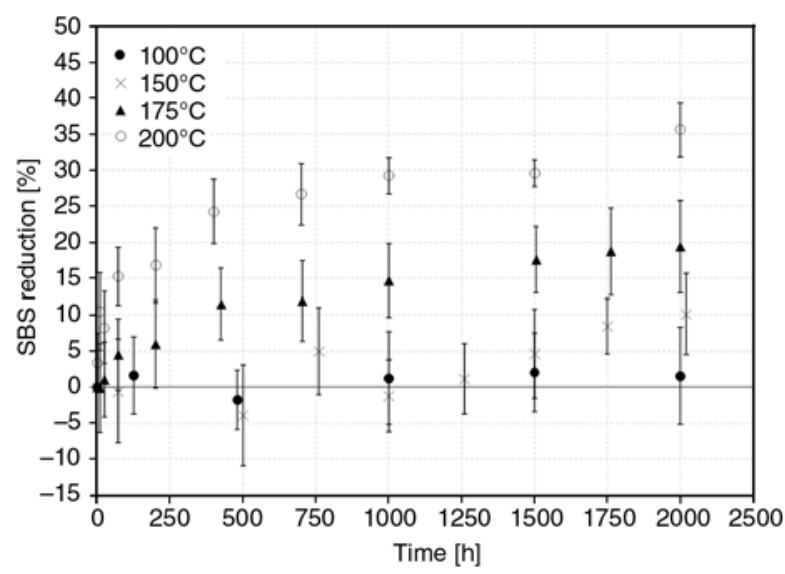

Figure 2. Percentage short beam strength reduction of the composite specimens subjected to isothermal thermo-oxidative aging at four different temperatures

Figure 2 illustrates the percent reduction of short beam strength at $100,150,175$, and $200^{\circ} \mathrm{C}$ up to 2000 hours of aging. To obtain the percent short beam strength reduction at different times, the measured strength values of eight aged samples were compared with those of the 27 unaged specimens that were tested earlier. The testing of 27 unaged specimens gave an initial SBS value of $87.06 \pm 2.8 \mathrm{MPa}$ at $95 \%$ confidence interval. At the start of the experiments, testing a much higher number of unaged samples was important to establish a reliable baseline value for SBS. It is obvious from Figure 2 that the variation of short beam strength within a sample set is much larger than the weight loss as indicated by the larger uncertainty bars. Considering that a new sample set is tested for each data point and the inherent uncertainty in characterizing strength of composites, the variation observed in Figure 2 is rather modest. Similar to the weight loss measurements, strength data is obtained at much shorter intervals during the initial phase of aging. By testing considerable number of samples within the first 250 hours, initial degradation rates are expected to be characterized sufficiently accurate for model development.

Samples aged at $100^{\circ} \mathrm{C}$ did not show a discernable strength reduction within the first 2000 hours. Unchanged strength values validate the earlier assessment that the initial weight loss observed in these samples were in fact due to the loss of moisture within the samples. Hence, data obtained at $100^{\circ} \mathrm{C}$ are not used in degradation models developed later. 
Samples aged at higher temperatures depict a welldefined strength reduction trend that steadily increases with time and temperature. After 2000 hours of aging, samples exposed to 150,175 and $200^{\circ} \mathrm{C}$ suffered approximately 10,20 and $35 \%$ reduction in short beam strength, respectively. It is also observed that composite laminates at $200^{\circ} \mathrm{C}$ experienced a much rapid degradation, suffering more than $10 \%$ strength loss within the first 24 hours. The same level of strength reduction is reached only after 400 and 2000 hours for samples exposed to 175 and $150^{\circ} \mathrm{C}$, respectively.

\subsection{Predictive modeling}

To develop predictive thermo-oxidative degradation models, the time rate of reduction of any composite property including its weight is chosen to be written in Equation (1):

$$
\frac{\mathrm{d} \phi}{\mathrm{d} t}=g(T, \phi)
$$

where $\phi$ is the degradation parameter and $T$ represents the temperature. In this study $\phi$ is defined to be either weight loss or SBS reduction; however it can be applied to capture the change of any other property. It is emphasized that in Equation (1), the functional form of $g(T, \phi)$ and thus, the specific formulation of temperature effect in $g(T, \phi)$ is not known a priori. Rather, it is left to be determined by the trend observed in experimental data. Once $g(T, \phi)$ is properly identified, the reduction in the property $\phi$ can be determined by solving a first order differential equation.

The premise in Equation (1) is that the thermooxidative degradation of composites can be expressed by a first order, phenomenological model. That is, temporal evolution of weight loss or SBS reduction during aging essentially exhibits a mathematical structure that can be predicted from a first order differential equation. Similar first order models have been used to describe oxidation reactions, where the function $g(T, \phi)$ can be separated as the product of two functions (i.e., $K(T) g(\phi)$ ). In addition to thermal aging, Equation (1), in this generic form, has been used to develop phenomenological models for various other applications, including cure modeling of thermoset resins and composite prepregs, and also for describing microstructural changes [18-22].
The first step in developing such a model involves construction of $\mathrm{d} \phi / \mathrm{d} t$ from the experimental data. The construction of this rate information requires data to be collected at sufficiently frequent intervals to form an accurate picture of the time rate of change of the degradation parameter, $\phi$. The subsequent construction of $\mathrm{d} \phi / \mathrm{d} t$ versus $\phi$ curves for the isothermal aging temperatures helps identify the suitable functional forms for $g(T, \phi)$.

In this study, the selection of the functional form of $g(T, \phi)$ and the other temperature dependent model parameters was dictated by the ability of the model to recover the isothermal aging data accurately. This was achieved by maximizing the correlation coefficient of the curve fit during the selection of model parameters and their dependence on temperature. By following this procedure, the best fit for the model parameters was obtained regardless of the scatter in the data.

Considering the data in Figure 1, a three-parameter model accurately represents the evolution of the percentage weight loss, $W_{l}$ (Equation (2)):

$$
\frac{\mathrm{d} W_{l}}{\mathrm{~d} t}=a \exp \left[b W_{l}^{c}\right]
$$

where $a, b$, and $c$ are the temperature dependent model parameters. These model parameters are expressed as a linear function of temperature (Equation (3)):

$$
\begin{aligned}
& a=5.64 \cdot 10^{-4} T-0.0908 \\
& b=0.0400 T-9.762 \\
& c=0.0148 T-2.260
\end{aligned}
$$

where $T$ represents aging temperature. For aging at $150^{\circ} \mathrm{C}$, a linear model seemed to work better as the weight loss increased slowly at a constant rate. Thus, instead of Equations (2)-(3), the linearized model, $W_{l}=A t+B$ where $A=1.314 \cdot 10^{-4}$ and $B=0.1648$ is more suitable. It can be easily seen that the initial rate of weight loss at $t=0$ is estimated by these models as $W_{l}=1.314 \cdot 10^{-4}, 0.0079$, $0.022 \%$ per hour for 150,175 and $200^{\circ} \mathrm{C}$, respectively.

In developing the short beam strength reduction model, a similar procedure is implemented to the data shown in Figure 2. The short beam strength of the specimens exposed to $100^{\circ} \mathrm{C}$ did not show a remarkable drop-off throughout the 2000 hours aging. Therefore, as in the weight loss model, 
$100^{\circ} \mathrm{C}$-data were excluded from the strength reduction model. In this case, a simpler two-parameter model for strength reduction worked better for all three temperatures. The time rate of reduction of strength is expressed by an exponential function given as Equation (4):

$$
\frac{\mathrm{d} \sigma}{\mathrm{d} t}=\kappa \exp (\lambda \sigma)
$$

where $\sigma$ is the percentage reduction in short beam strength, and $\kappa$ and $\lambda$ are temperature dependent model parameters. Within the range of 150 to $200^{\circ} \mathrm{C}$, the parameters $\kappa$ and $\lambda$ were expressed in Equation (5):

$$
\begin{aligned}
& \kappa=\left(1.256 \cdot 10^{-3}\right) T^{2}-(0.405) T+32.569 \\
& \lambda=\left(1.895 \cdot 10^{-5}\right) T^{2}-\left(8.327 \cdot 10^{-3}\right) T+0.712
\end{aligned}
$$

Although the model given in Equations (4)-(5) only contains two parameters, $\kappa$ and $\lambda$, the temperature dependency of these parameters did not yield a linear relationship. The model estimates the initial rates of short beam strength loss at $t=0$ as 0.079 , 0.159 , and $1.809 \%$ per hour for 150,175 and $200^{\circ} \mathrm{C}$, respectively. These initial rates are approximate values obtained for $t \rightarrow 0$. As time increases, the strength reduction rates rapidly change, especially for higher temperatures. Nevertheless, the estimates of strength reduction as $t \rightarrow 0$ provide valuable guideline regarding the severeness of the initial degradation.

\subsection{Long-term predictions and model validation}

The applicability of the weight loss and short beam strength reduction models to longer time frames was analyzed. Although these models have been developed by using only 2000 hours of aging data, applying them to longer periods is desirable. Such an approach would save significant time in experimental characterization of composite degradation, provided that the desired accuracy is still maintained. In the present study, extending the time frame of the models by 2.5 times from 2000 to 5000 hours will demonstrate the utility and robustness of the models. To validate this approach, predictions of both models for 5000 hours aging are compared with the actual experimental data.
The weight loss model given by Equations (2)-(3) is numerically solved up to 5000 hours. The solution is rather straight forward since the standard fourth-order Runge-Kutta method yields the desired numerical accuracy. Figure 3 shows the experimental and predicted percentage weight loss up to 5000 hours. The solid lines illustrate the model predictions up to 2000 hours, the time frame used in model development. Dashed lines depict extension of model predictions up to 5000 hours. As explained earlier, the samples aged at $100^{\circ} \mathrm{C}$ did not suffer any weight loss beyond the initial phase of moisture desorption. The model performed very well for samples aged at 150 and $175^{\circ} \mathrm{C}$, with weight loss estimates falling almost within the measured experimental uncertainty. It seems that at 150 and $175^{\circ} \mathrm{C}$, the model would continue to predict weight loss accurately, even after 5000 hours. For aging at $200^{\circ} \mathrm{C}$, the model, however, loses accuracy, such that the experimental data starts diverging from the established trend shortly after 2500 hours. At 5000 hours, the model predicts a weight loss of $4 \%$, whereas experimental data indicates an almost $6 \%$ weight loss. It is worth noting that experimental uncertainty increases considerably after 2500 hours, as observed by the continual increase in the size of the error bars up until 5000 hours. This deviation from the initial trend points to an acceleration in degradation mechanism.

Although, the reasons for this acceleration in degradation have not been identified, it could possibly be

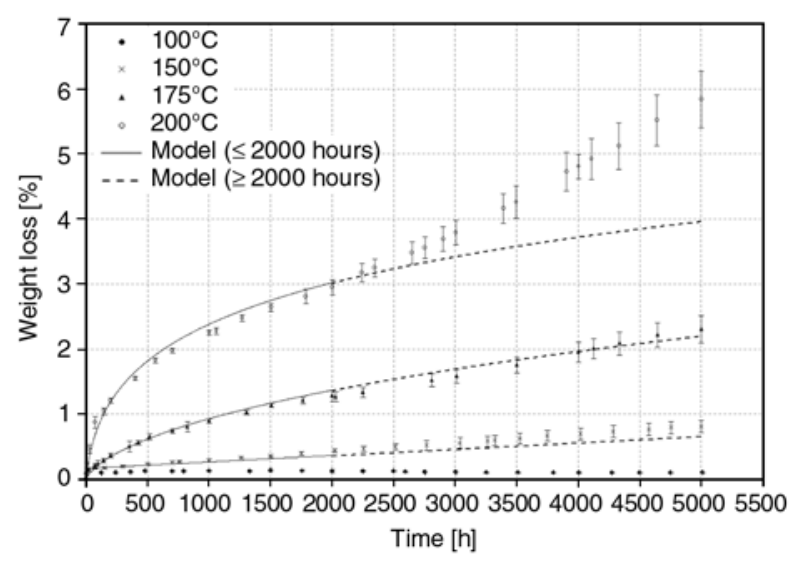

Figure 3. Experimental and predicted percentage weight loss of the composite specimens subjected to thermo-oxidative aging up to 5000 hours. Solid lines represent the predictions within the first 2000 hours for which the models were developed. Dashed line represents the extension of model predictions up to 5000 hours. 
due to further weakening of the overall fiber-matrix interface, severe delamination or acceleration of microcrack growth along the fibers at the interphase region. A detailed microscopic examination of the samples was not performed to establish the leading cause of degradation at higher temperatures; however, the surface morphology of the samples displayed visible changes such as charring and discoloration after being exposed to $200^{\circ} \mathrm{C}$ for 5000 hours. From a modeling perspective, higher order effects that were not captured by the first order models may be becoming dominant at later stages of degradation. Such effects could be included in the models at the expense of adding mathematical complexity. For example, both reaction and diffusion controlled degradation effects could be captured by a combination of both first and second order temporal differentials.

Figure 4 shows the experimental and predicted percent reduction in short beam strength up to 5000 hours. Samples aged at $100^{\circ} \mathrm{C}$ did not suffer any identifiable strength loss as expected. Model predictions and experimental results for 150, 175 and $200^{\circ} \mathrm{C}$ are observed to agree very well, with the vast majority of model estimates falling within the range of experimental uncertainty. Model performance for aging at $150^{\circ} \mathrm{C}$ has been excellent, where the composite samples suffer a $10.8 \%$ strength reduction after 5000 hours. Model predictions also show excellent agreement up to 4000 hours for aging at $175^{\circ} \mathrm{C}$ where the samples record $24.9 \%$

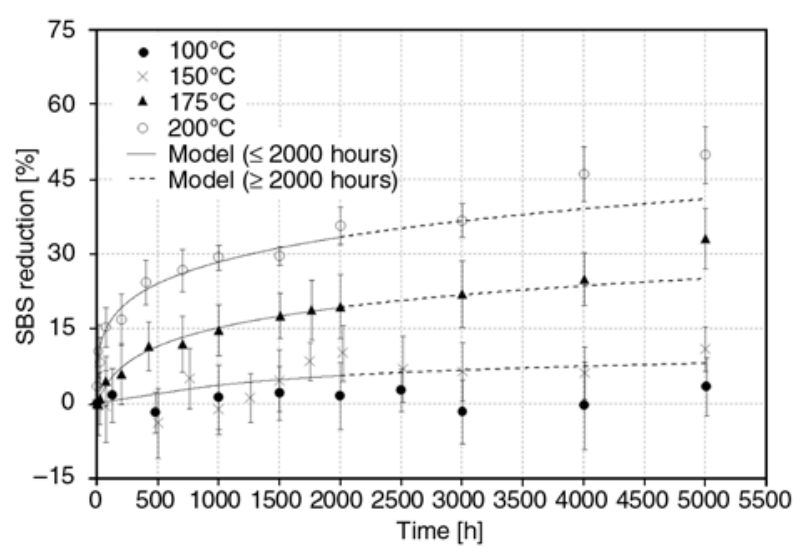

Figure 4. Experimental and predicted percentage short beam strength reduction of the composite specimens subjected to thermo-oxidative aging up to 5000 hours. Solid lines represent the predictions within the first 2000 hours for which the models were developed. Dashed line represents the extension of model predictions up to 5000 hours. strength reduction. These results indicate that although the material system AS4/3501-6 has been developed for use up to $177^{\circ} \mathrm{C}$, exposure to temperatures as low as $150^{\circ} \mathrm{C}$ for longer periods could lead to delamination failure due to interlaminar shear stresses. Therefore, it is essential to account for the thermo-oxidative degradation in estimating service life if the composite product is to be exposed to elevated temperatures.

For $200^{\circ} \mathrm{C}$, the model predictions are excellent up to 3000 hours, where the samples record $36.7 \%$ strength reduction. The accelerated pace of weight loss observed at $200^{\circ} \mathrm{C}$ did not lead to a similar rapid increase in the strength loss. There is some deviation observed in model predictions for $200^{\circ} \mathrm{C}$ at 4000 and 5000 hours. However, at these levels, the samples have already lost more than $45 \%$ of their initial strength. In most practical applications, these samples would be viewed as failed - if not already failed in service - and therefore would be replaced before reaching such excessive levels of degradation. Overall, the strength loss model performed very well for all the temperatures and well beyond the time frame for which it was developed.

\section{Conclusions}

Thermo-oxidative aging of 24-ply, aerospace-grade AS4/3501-6 graphite/epoxy composite laminates is investigated by characterizing the weight loss and reduction in short beam strength at 100, 150, 175 and $200^{\circ} \mathrm{C}$. First order differential models are developed for the percentage weight loss and short beam strength reduction using the isothermal aging data up to 2000 hours. These models can be used to predict thermo-oxidative aging when the composite is exposed to an arbitrary time-dependent temperature profile. The utility of these models were demonstrated by successfully predicting the actual weight loss and strength reduction up to 5000 hours, well beyond 2000 hours for which the models were developed. Thus, it is possible to develop robust models using shorter term, isothermal aging data which would yield sufficiently accurate results over a much longer time frame.

The material was shown to be highly stable at $100^{\circ} \mathrm{C}$ such that no significant weight loss or strength reduction was observed during 5000 hours of aging. After 5000 hours, the laminates suffered $0.81,2.31$, and $5.84 \%$ weight loss coupled with 
$10.87,33.07$, and $49.86 \%$ short beam strength reduction at 150,175 , and $200^{\circ} \mathrm{C}$, respectively. Compared to aging at 150 and $175^{\circ} \mathrm{C}$, aging at $200^{\circ} \mathrm{C}$ led to rapid strength reduction of more than $20 \%$ within the first 400 hours. Overall, the weight loss and strength reduction models predicted data accurately, particularly when the weight loss is less than $3 \%$ and the strength reduction is less than $35 \%$.

\section{References}

[1] Colin X., Verdu J.: Thermal ageing and lifetime prediction for organic matrix composites. Plastics, Rubber and Composites, 32, 349-356 (2003). DOI: $10.1179 / 146580103225004117$

[2] Tsotsis T. K., Keller S., Bardis J., Bish J.: Preliminary evaluation of the use of elevated pressure to accelerate thermo-oxidative aging in composites. Polymer Degradation and Stability, 64, 207-212 (1999). DOI: 10.1016/S0141-3910(98)00190-6

[3] Burcham L. J., Eduljee R. F., Gillespie Jr. J. W.: Investigation of the microcracking behavior of bismaleimide composites during thermal aging. Polymer Composites, 16, 507-517 (1995). DOI: $\underline{10.1002 / p c .750160609}$

[4] Greer R. H.: Thermal aging of contemporary graphite/ epoxy materials. in 'Proceedings of $24^{\text {th }}$ International SAMPE Symposium and Exhibition. San Francisco, USA' 1039-1052 (1979).

[5] Nelson J. B.: Thermal aging of graphite/polyimide composites. in: 'Long-term behavior of composites' (ed.: O’Brien T. K.) ASTM STP 813, Philadelphia, 206-221 (1983).

[6] Bowles K. J., Meyers A.: Specimen geometry effects on graphite/PMR-15 composites during thermooxidative aging. in 'Proceedings of $31^{\text {st }}$ International SAMPE Symposium and Exhibition. Las Vegas, USA' 1285-1299 (1986).

[7] McManus H. L., Foch B. J., Cunningham R. A.: Mechanism-based modeling of long-term degradation. Journal of Composites Technology and Research, 22, 146-152 (2000).

[8] Salin I. M., Seferis J. C., Loechelt C. L., Rothschilds R.: Time-temperature equivalence in thermogravimetry for BMI composites. SAMPE Quarterly, 24, 54-62 (1992).

[9] Seferis J. C.: Aging analyses of polymer composites through time-temperature equivalence. Journal of Composites Technology and Research, 21, 173-179 (1999).

[10] Chung K., Seferis J. C., Nam J-D.: Investigation of thermal degradation behavior of polymeric composites: Prediction of thermal cycling effect from isothermal data. Composites Part A: Applied Science and Manufacturing, 31, 945-957 (2000). DOI: $\underline{10.1016 / \mathrm{S} 1359-835 \mathrm{X}(00) 00043-9}$
[11] Tsotsis T. K., Keller S., Lee K., Bardis J., Bish J.: Aging of polymeric composite specimens for 5000 hours at elevated pressure and temperature. Composites Science and Technology, 61, 75-86 (2001). DOI: $10.1016 / \mathrm{S} 0266-3538(00) 00196-2$

[12] Rasoldier N., Colin X., Verdu J., Bocquet M., Olivier L., Chocinski-Arnault L., Lafarie-Frenot M. C.: Model systems for thermo-oxidised epoxy composite matrices. Composites Part A: Applied Science and Manufacturing, 39, 1522-1529 (2008).

DOI: 10.1016/j.compositesa.2008.05.016

[13] Dao B., Hodgkin J., Krstina J., Mardel J., Tian W.: Accelerated aging versus realistic aging in aerospace composite materials. II. Chemistry of thermal aging in a structural composite. Journal of Applied Polymer Science, 102, 3221-3232 (2006).

DOI: $10.1002 /$ app. 24573

[14] Kim J., Moon T. J., Howell J. R.: Cure kinetic model, heat of reaction, and glass transition temperature of AS4/3501-6 graphite-epoxy prepregs. Journal of Composite Materials, 36, 2479-2498 (2002). DOI: $10.1177 / 0021998302036021712$

[15] Kim Y. K., White S. R.: Stress relaxation behavior of 3501-6 epoxy resin during cure. Polymer Engineering and Science, 36, 2852-2862 (1996).

DOI: $10.1002 /$ pen. 10686

[16] Hou T. H., Bai J. M.: A semi-empirical approach for the chemoviscosity modeling of reactive resin system. SAMPE Journal, 24, 43-51 (1988).

[17] Howsmon S. C.: Viscoelastic and mechanical properties of Hercules 3501-6 epoxy resin and its prepreg. Master's thesis, The University of Oklahoma (2005).

[18] Loos A. C., Springer G. S.: Curing of epoxy matrix composites. Journal of Composite Materials, 17, 135169 (1983). DOI: $10.1177 / 002199838301700204$

[19] Hargis M., Grady B. P., Aktas L., Bomireddy K. R., Howsmon S., Altan M. C., Rose T., Rose H.: Calorimetric and rheological measurements of three commercial thermosetting prepreg epoxies. Journal of Composite Materials, 40, 873-897 (2006). DOI: $\underline{10.1177 / 0021998305056380}$

[20] Wetzel E. D., Don R. C., Gillespie Jr. J. W.: Modeling thermal degradation during thermoplastic fusion bonding of thermoset composites. in 'Proceedings of Society of Plastics Engineers 52 ${ }^{\text {nd }}$ Annual Technical Conference (ANTEC '94). San Francisco, USA' 1263-1268 (1994).

[21] Nam J-D., Seferis J. C.: Generalized composite degradation kinetics for polymeric systems under isothermal and nonisothermal conditions. Journal of Polymer Science Part B: Polymer Physics, 30, 455-463 (1992). DOI: $10.1002 /$ polb.1992.090300505

[22] Hancox N. L.: Thermal effects on polymer matrix composites: Part 2. Thermal degradation. Materials and Design, 19, 93-97 (1998). DOI: $\underline{10.1016 / \mathrm{S} 0261-3069(98) 00019-3}$ 